Check for updates

Cite this: Phys. Chem. Chem. Phys., 2020, 22, 3264

Received 10th December 2019, Accepted 16th January 2020

DOI: $10.1039 / c 9 c p 06661 f$

rsc.li/pccp

\section{Proton dynamics in molecular solvent clusters as an indicator for hydrogen bond network strength in confined geometries $\dagger$}

\author{
Clara-Magdalena Saak, (D) a Clemens Richter, (D) ${ }^{\mathrm{b}}$ Isaak Unger, ${ }^{\mathrm{a}}$ Melanie Mucke, ${ }^{\mathrm{a}}$ \\ Christophe Nicolas, ${ }^{C}$ Uwe Hergenhahn, (iD $\ddagger^{\text {bd }}$ Carl Caleman, (D) ae Marko Huttula, ${ }^{\dagger}$ \\ Minna Patanen (iD ${ }^{f}$ and Olle Björneholm*a
}

\begin{abstract}
Hydrogen bonding leads to the formation of strong, extended intermolecular networks in molecular liquids such as water. However, it is less well-known how robust the network is to environments in which surface formation or confinement effects become prominent, such as in clusters or droplets. Such systems provide a useful way to probe the robustness of the network, since the degree of confinement can be tuned by altering the cluster size, changing both the surface-to-volume ratio and the radius of curvature. To explore the formation of hydrogen bond networks in confined geometries, here we present $O$ 1s Auger spectra of small and large clusters of water, methanol, and dimethyl ether, as well as their deuterated equivalents. The Auger spectra of the clusters and the corresponding macroscopic liquids are compared and evaluated for an isotope effect, which is due to proton dynamics within the lifetime of the core hole (proton-transfer-mediated charge-separation, PTM-CS), and can be linked to the formation of a hydrogen bond network in the system. An isotope effect is observed in water and methanol but not for dimethyl ether, which cannot donate a hydrogen bond at its oxygen site. The isotope effect, and therefore the strength of the hydrogen bond network, is more pronounced in water than in methanol. Its value depends on the average size of the cluster, indicating that confinement effects change proton dynamics in the core ionised excited state.
\end{abstract}

\section{Introduction}

In molecular terms, macroscopic liquids are often described as 'infinite', meaning their bulk intermolecular structure is free from modulation by boundary structures imposed by the formation of a surface or interface. In hydrogen bonded liquids such as water, this allows the bulk molecules to realise strong extended hydrogen bond networks. However, it is less well-known how robust the hydrogen bond network is to perturbations which

\footnotetext{
${ }^{a}$ Department for Physics and Astronomy, Uppsala University, Box 516, 75120 Uppsala, Sweden. E-mail: olle.bjorneholm@physics.uu.se

${ }^{b}$ Leibniz Institute of Surface Engineering (IOM), Permoserstr. 15, 04318 Leipzig, Germany

${ }^{c}$ Synchrotron SOLEIL, L'Orme des Merisiers, Saint-Aubin, BP 48, Gif-sur-Yvette, Cedex, 91192, France

${ }^{d}$ Fritz-Haber-Institut der Max-Planck-Gesellschaft, Faradayweg 4-6, 14195 Berlin, Germany

${ }^{e}$ Center for Free-Electron Laser Science, Deutsches Elektronen-Synchrotron, Notkestr. 85, 22607 Hamburg, Germany

${ }^{f}$ Nano and Molecular Systems Research Unit, Faculty of Science, University of Oulu, P. O. Box 3000, 90014, Finland

$\dagger$ Electronic supplementary information (ESI) available. See DOI: 10.1039/c9cp06661f

\$ Max Planck Institute for Plasma Physics, 17491 Greifswald, Germany.
}

disrupt the 'infinite' structure, for example by the formation of a surface/interface, or confinement effects caused by the formation of small clusters and droplets. Surface structure is especially important in small clusters or droplets, since they have a high surface-to-volume ratio, which increases the proportion of molecules residing at the surface with a lower hydrogen bond coordination number than molecules in the bulk. In water, at least 25 molecules are needed before the cluster can contain a fully-enclosed 'bulk-like' molecule. ${ }^{1}$ In addition, their spherical geometry can also introduce so-called confinement effects which alter the intermolecular structure differently from the formation of a planar surface. The smaller the radius of curvature, the more additional strain is put on the interactions between the surface molecules. Even the bulk molecules can be affected by the constrained cluster geometry, since they are compressed by an internal pressure, affecting the formation of tetrahedral hydrogen bond structures.

It also remains relatively unexplored how confinement effects influence the hydrogen bond network in other solvents that have intrinsically weaker hydrogen bond networks, and are thus potentially better able to accommodate structural deformations due to the less rigid intermolecular networks. 
The molecular differences between water and methanol lead their respective surface structures to be very different, as described by Wilson et $a .^{2}$ For water, the authors found an expansion of the intermolecular $\mathrm{O}-\mathrm{O}$ distance at the surface compared with the bulk. In addition, the water surface has also been found to contain a significant fraction of molecules with unsaturated hydrogen bond sites, such as dangling $\mathrm{OH}$ bonds, and acceptor-only molecules. ${ }^{3}$ The molecules residing at the surface also display increased diffusion compared with the bulk. ${ }^{4}$ Both the decreased coordination number and the increased average $\mathrm{O}-\mathrm{O}$ distance indicate a weakening of the water hydrogen bond network at the surface, compared with the bulk.

In methanol, on the other hand, Wilson et $a .^{2}$ found a contraction of the $\mathrm{O}-\mathrm{O}$ distances in the surface layer. Sum frequency generation (SFG) experiments ${ }^{5,6}$ have shown that at the surface of methanol the hydrophobic expulsion of the methyl group leads to a more ordered structure, with the methyl group facing towards the vacuum side of the surface and the hydroxyl group towards the bulk. This arrangement decreases steric hindrance at the surface and increases hydrogen bonding. Due to the increase in molecular order and hydrogen bonding the methanol surface is sometimes referred to as "ice-like".

These differences in the way the hydrogen bond network of water and methanol changes its structure at the surface might also be related to the two liquids' different surface tensions.

In clusters, the curvature of the surface produces additional strain on the molecular structure, with the radius of curvature decreasing with decreasing cluster size. This leads to additional restructuring at the surface, likely weakening the intermolecular interactions, as it introduces further restrictions on the available surface geometries.

Here, we explore experimentally the question of how confinement effects in solvent clusters affect the overall strength and robustness of the hydrogen bond network. We present soft $\mathrm{X}$-ray photoelectron spectroscopy (XPS) and O 1s Auger data for water $\left(\mathrm{H}_{2} \mathrm{O}\right)$, methanol $(\mathrm{MeOH})$ and dimethyl ether (DME) clusters and compare these systems to the bulk liquid of water and methanol. While a variation of the cluster size allows for an investigation of the degree of confinement, the choice of molecules provides hydrogen bond networks of decreasing strength with progressive degrees of methylation. Data were also recorded for the equivalent deuterated systems, and the Auger spectra were evaluated for an isotope-dependent change in the shape of the Auger spectrum, which can be attributed to proton dynamics within the lifetime of the $\mathrm{O} 1 \mathrm{~s}$ core hole. ${ }^{7}$ The changed dynamics in the excited state are then related to a change in the overall strength of hydrogen bonding in the system. ${ }^{8}$

\section{Experimental}

The cluster hydrogen bond networks were studied by examining the $\mathrm{O}$ 1s Auger electron spectra. Here, we focus specifically on the contribution of proton dynamics along a hydrogen bond in the core ionised state to the total signal distribution in the O 1s Auger region. In the case of liquid water, proton transfer during the lifetime of the $\mathrm{O} 1 \mathrm{~s}^{-1}$ intermediate state has been studied in detail by Thürmer et $a .^{7}$ and in subsequent publications. ${ }^{8-10}$ The mechanism outlined in the original paper, termed proton-transfer-mediated-charge-separation (PTM-CS), is briefly summarised below.

When a molecule is core ionised, it is left in a highly excited state $\left(\mathrm{O} 1 \mathrm{~s}^{-1}\right)$. This state can decay by refilling the core-hole with a valence electron. The excess energy can then be dissipated by the emission of a high energy photon, or by emitting another valence electron (Auger electron) which leaves the system in a doubly valence-ionised final state. In this work, we only focus on processes that lead to the emission of a secondary Auger electron, since $>99 \%$ of core holes will relax via this channel. ${ }^{11}$ The lifetime of the intermediate excited state is determined by the core-hole lifetime of the $\mathrm{O} 1 \mathrm{~s}$, which is approximately $4 \mathrm{fs}^{12}$ Within this lifetime the heavy atomic cores can be considered to be frozen, however some proton dynamics may occur. In the gas phase, such effects have previously been studied under the term ultrafast dissociation. ${ }^{13}$

If the electronic decay to the final state occurs in a (partially-) proton transferred geometry, the loss of the proton decreases the total final charge on the ionised molecule. This separation of the charge over two molecules lowers the energy of the final state significantly, which in turn increases the excess energy carried by the secondary electron. The PTM-CS effect therefore leads to an increase in the intensity of the high kinetic energy flank of the Auger spectrum. Proton transfer in the excited state can also occur in concert with non-local decay channels such as 'interatomic coulombic decay' (ICD) ${ }^{14-17}$ and 'electron transfer mediated decay' (ETMD). ${ }^{18,19}$ In these cases the transfer of a proton affects the energy of the final state to a lesser degree, since the non-local decay channels already lead to a final state in which the total charge $(2+)$ is shared between two molecules.

To isolate the PTM-CS feature from the other decay channels, deuterium is substituted for hydrogen in the system; the heavier isotope leads to a decreased dispersion of the wave packet in the core ionised excited state, i.e. the deuterium atom does not migrate to the same degree as the proton, and a higher percentage of states decay close to the ground state geometry. Both local and non-local decay processes occurring in the ground state geometry, without any proton dynamics taking place, are not affected by the isotopic substitution since the geometry of the system is not significantly altered in terms of intermolecular distances. We can therefore isolate the PTM-CS feature, which is caused by the dynamics of the proton, by comparing measurements of the light/normal system and its deuterated equivalent to obtain a difference trace showing the relative redistribution of signal intensity due to proton transfer.

It is particularly interesting that the $\mathrm{O} 1 \mathrm{~s}^{-1}$ state in hydrogen bonded systems, such as water, is not dissociative in the gas phase, but dissociation is allowed in the hydrogen bonded condensed phase. ${ }^{20}$ The occurrence of proton transfer dynamics within the lifetime of the $\mathrm{O} 1 \mathrm{~s}$ core hole was therefore linked to hydrogen bonding.

Proton transfer in the core ionised excited state can only occur if the potential curve of the state is dissociative. The $\mathrm{O} 1 \mathrm{~s}^{-1}$ states 
of water and methanol have been shown to be non-dissociative in the gas phase, ${ }^{7,21}$ which means that the interaction with the surrounding molecules, that takes place in the condensed phase, reshapes the potential to the extent that the state becomes dissociative, i.e. the channel for proton dynamics opens when the ionised molecule is engaged in sufficiently strong interaction with the surrounding molecules. In protic systems such as water and methanol, the strongest intermolecular interactions are hydrogen bonds, and we therefore assume that the changes in the excited state potential are mainly due to the formation of hydrogen bonds. The connection of nuclear and electronic motion that occurs during the PTM-CS process has been discussed in detail by Slavíček et $a l^{8}{ }^{8}$ Another argument for the importance of the hydrogen bond in facilitating the dissociation process is that the proton will initially move through a Zundel-like structure, which is characterised by an elongated O-H bond. A sufficient electron density $\left(\delta^{-}\right)$close to the proton is therefore required to stabilise the positive charge site created by the dissociation event. Formation of a hydrogen bond in the ground state brings the molecules into a geometry in which the $\mathrm{O}-\mathrm{H}$ bond and the lone-pair are arranged such that the proton can be transferred in the excited state.

In the case of deuterium substitution, the state remains dissociative, however, the larger mass of the isotope leads to a slower dispersion of the wavepacket along the proton transfer axis, leading to different degrees of (partial) proton transfer in the two isotopomers.

The hydrogen bonding network can influence the experimentally observed isotope effect in different ways: in a system of equally strong hydrogen bonds the number of hydrogen bonds will determine the fraction of oxygen sites which can undergo dissociation in the excited state. The magnitude of the isotope effect is therefore proportional to the number of hydrogen bonds formed in the system. The hydrogen bonds found in a liquid or amorphous system are, however, of varying strength and it is reasonable to assume that a strong hydrogen bond affects the shape of the excited state potential more strongly than a weak interaction. The steepness of the dissociative potential is therefore influenced by the hydrogen bond strength. This difference in the shape of the excited state potential will in turn affect the dispersion of the wave packets and the magnitude of the experimentally observed isotope effect. Our experiment therefore represents a convolution of the number of hydrogen bonds and their respective strength. Since the hydrogen bonding found in associated liquids such as water is highly cooperative and cannot simply be described as the sum of two-body interactions, ${ }^{22}$ we attribute the isotope effect to the average strength of the hydrogen bond network, rather than an individual bond strength and number.

Measurements were conducted at the PLÉIADES beamline situated at the synchrotron light source SOLEIL in Paris, France. The experimental setup consists of a multipurpose source chamber and a main chamber, on which a hemispherical electron analyser is mounted - a detailed description of the experimental endstation is given in a publication by Lindblad et $a .^{23}$ and a sketch of the experimental setup is shown in Fig. 1. The cluster

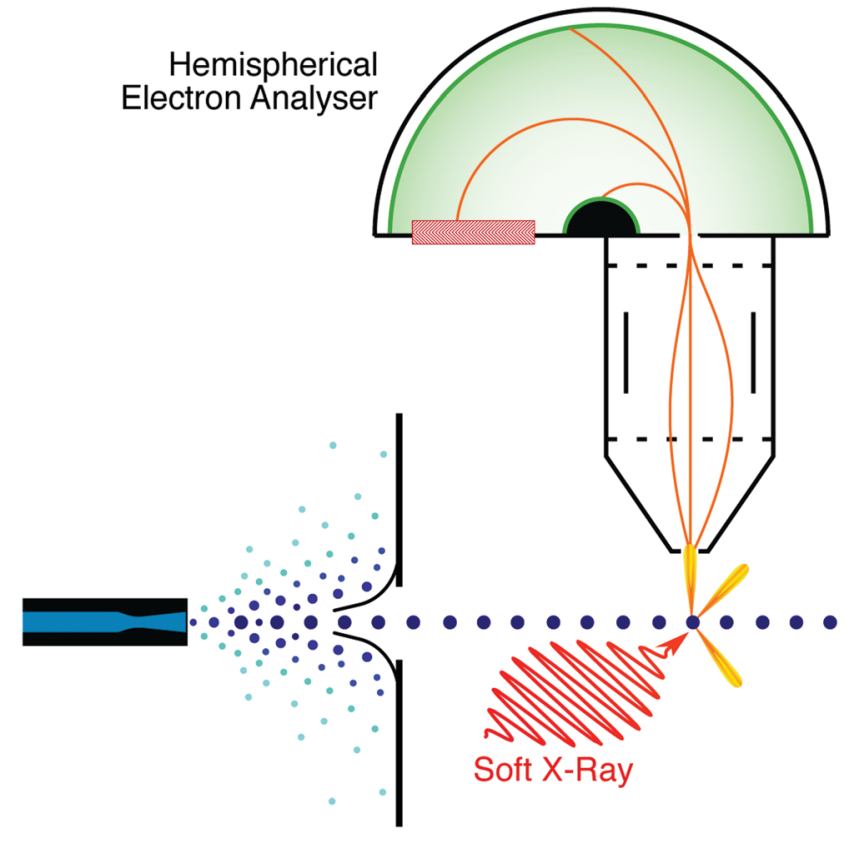

Supersonic expansion

Spectrometer chamber

Fig. 1 Schematic of the experimental setup at the PLÉIADES beamline (SOLEIL) used for cluster experiments. In the case of the liquid measurements at BESSY the experimental geometry is very similar, the only major difference is that the supersonic expansion chamber is replaced by a liquid jet. A more detailed description of the experiment can be found in the main text.

distribution is produced via continuous supersonic expansion of the sample vapour. In the case of water and methanol experiments, the sample was placed in a heated reservoir and $\mathrm{N}_{2}$ carrier gas was bubbled through the liquid, producing a mixture of $\mathrm{N}_{2}$ /solvent vapour which was expanded through a nozzle. Gaseous DME was mixed with He carrier gas in a separate vessel with a 2 bar DME and 6 bar He mixing ratio. The cluster nozzle has a converging/diverging shape with a diameter of $100 \mu \mathrm{m}$ at the narrowest point and its temperature can be controlled with Peltier elements. The average size of the cluster distribution was varied by adjusting the temperature of the nozzle and the liquid reservoir, as well as the pressure of the carrier gas; the experimental conditions used to produce the different cluster distributions are given in Table 1 . The cluster distribution enters the main chamber through a skimmer (beam-dynamics, $400 \mu \mathrm{m}$ ) where it is perpendicularly intersected with linearly polarised soft X-ray radiation at a photon energy of $600 \mathrm{eV}$.

To estimate the average size of the cluster distribution the binding energy (BE) shift of the cluster valence peak relative to the gas phase was determined and compared with literature data. The magnitude of this shift increases proportionally to the average size of the cluster distribution, ${ }^{24,25}$ thus allowing for a rough estimation of average cluster size. Scaling laws that predict the average cluster size based on the expansion conditions, such as those used for rare gas expansions ${ }^{26,27}$ or modified scaling laws for solvent clusters, ${ }^{28}$ cannot be used to estimate the average cluster size here since the sample is expanded with a carrier gas 
Table 1 Summary of cluster conditions used in this work. The average cluster sizes were estimated by comparing binding energy shifts with literature values, ${ }^{25,31}$ and should be seen as indicative of the order of magnitude of the average size in the cluster distribution, rather than the absolute size. The average cluster size was rounded to the nearest hundreds of molecules

\begin{tabular}{lllc}
\hline & & $\begin{array}{l}\text { Valence peak } \\
\text { BE shift }[\mathrm{eV}]\end{array}$ & $\begin{array}{l}\text { Estimated average } \\
\text { cluster size }[\langle n\rangle]\end{array}$ \\
\hline $\mathrm{H}_{2} \mathrm{O}$ & Small & 0.9 & 100 \\
& Large & 1.2 & $>600$ \\
$\mathrm{D}_{2} \mathrm{O}$ & Small & 0.9 & 100 \\
& Large & 1.1 & 600 \\
$\mathrm{MeOH}$ & Small & 0.9 & 100 \\
& Large & 1.1 & 600 \\
$\mathrm{~d}_{4}-\mathrm{MeOD}$ & Small & 0.9 & 100 \\
& Large & 1.0 & 300 \\
$\mathrm{DME}$ & & 0.8 & 100 \\
$\mathrm{~d}_{6}-\mathrm{DME}$ & & 0.9 & 300
\end{tabular}

which changes the cluster formation process during expansion into vacuum. The use of the binding energy shift, which we use here as an indicator of average cluster size, can only provide a rough estimate and therefore the average cluster sizes given here are rounded to the nearest hundreds of molecules.

The liquid phase measurements were obtained from a liquid jet target at the $\mathrm{SOL}^{3}$ endstation ${ }^{29}$ located at the U49-2/PGM1 beamline $^{30}$ of the BESSY II storage ring, Helmholtz-Zentrum Berlin. The liquid data of methanol shown here has previously been published; ${ }^{21}$ the water spectra were obtained in the same manner and a detailed report of the experimental method can be found in the publication on methanol. ${ }^{21}$ Since the spectra from a liquid jet target contain significant contributions from the surrounding vapour, a gas phase spectrum was subtracted from these traces to yield the so-called liquid-only spectra.

To allow for comparison between different systems, all Auger spectra are shifted in kinetic energy to align their main Auger feature with the main liquid water peak. The spectra are then normalised to their total area between 493 and $517 \mathrm{eV}$ kinetic energy, and this normalisation window around the main peak of the spectra is indicated in Fig. 2 and 4 by two grey dashed lines.

In our study, we use the area underneath the difference function of the normalised Auger traces measured for the normal and deuterated clusters and liquids we have investigated as a measure for the propensity of the PTM-CS channel. To quantify the experimental error in the value of these quantities we fit a smooth function composed of the sum of two Gaussian peaks in the case of water and four Gaussian functions in the case of methanol to each difference trace. The Gaussian peaks were chosen to represent different regions of relative intensity gain and loss in the difference spectrum, but no one-to-one correspondence with the actual Auger final states has been attempted or is claimed. The distribution functions of the residuals of these fits are shown in the ESI. $\dagger$ For each of them, we have determined its width $\sigma$. We then have determined the smallest and largest area difference compatible with the data by subtracting or adding $\sigma$ to the respective fitted difference function, using signs such that the integrated area is maximised

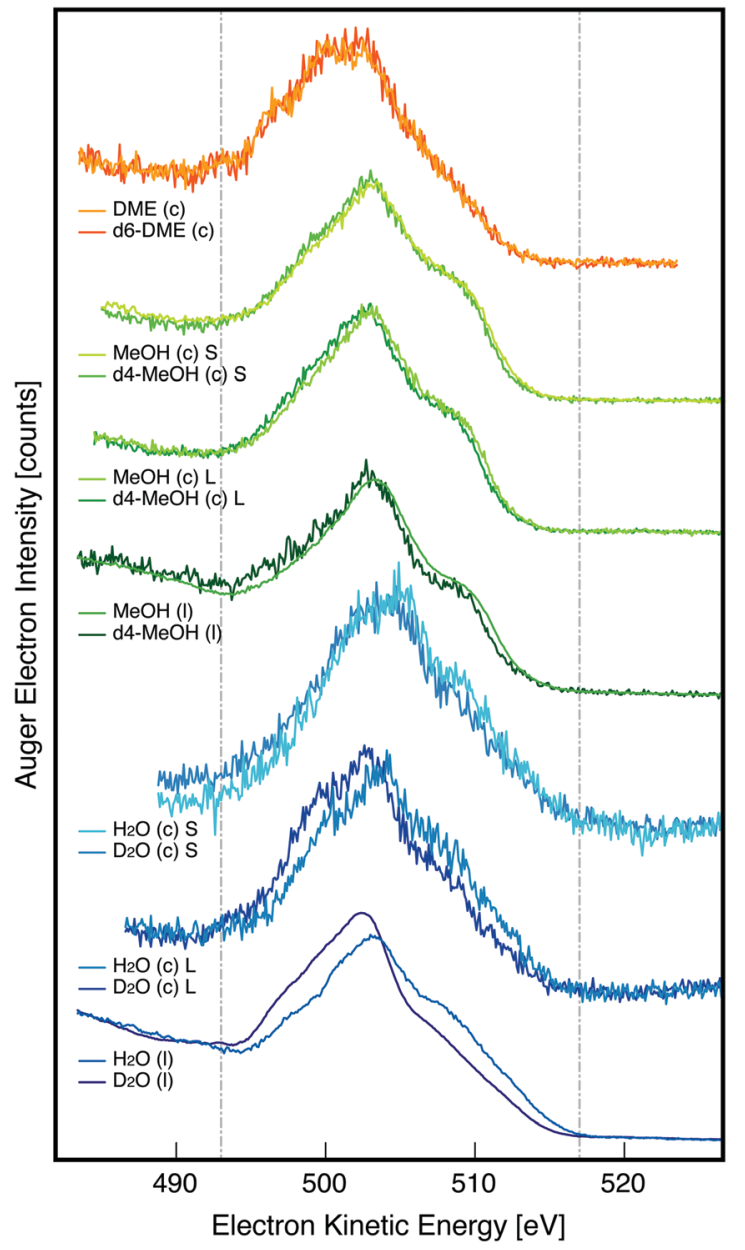

Fig. 2 Auger $\mathrm{O} 1 \mathrm{~s}$ spectra of water $\left(\mathrm{H}_{2} \mathrm{O}\right)$, methanol $(\mathrm{MeOH})$, and dimethyl ether (DME) clusters, as well as their fully deuterated equivalents. For water and methanol, the equivalent spectra for the bulk liquid are also shown. The labels (c) and (l) are used to indicate cluster and liquid data respectively. The small and large cluster conditions are indicated by $\mathrm{S}$ and L. Liquid methanol data has previously been published. ${ }^{21}$ All spectra were shifted in kinetic energy to align with the main peak of liquid water.

or minimised, respectively. Finally, all area differences and their upper and lower bounds were normalised to the water case, yielding the error bars shown in the Results section.

\section{Results and discussion}

Photoelectron measurements of the $\mathrm{O} 1 \mathrm{~s}$, O 1s Auger and valence band regions of two different size regimes for clusters of water and methanol, and one cluster size distribution of DME, as well as equivalent data for the fully deuterated systems were recorded. We estimate the average size of the studied cluster distributions via the binding energy shift between the valence peak of the gas phase and maximum of the corresponding cluster valence band, which are then compared with literature values. The binding energy shift of the individual cluster conditions are summarised in Table 1 . In water/heavy water we find the cluster phase to be shifted by $0.9 / 0.85 \mathrm{eV}$ for the small cluster conditions, and 1.15/1.05 eV for the large clusters, respectively. 
The two methanol cluster conditions produce a binding energy shift of $0.9 / 0.85 \mathrm{eV}$ and 1.05/1 eV at small and large cluster conditions, respectively, while for dimethyl ether clusters a binding energy shift of $0.8 / 0.9 \mathrm{eV}$ is observed.

Literature values from photoelectron spectroscopy giving the correlation between cluster-to-monomer binding energy shift and average cluster size exist for water ${ }^{31}$ and dimethyl ether, ${ }^{25}$ but not for methanol, to the best of our knowledge. Therefore, we have used the values determined for water to estimate the methanol cluster sizes. This likely leads to an underestimation of the size of these clusters since the binding energy shifts of water clusters are known to converge to bulk values at smaller sizes than other molecules. ${ }^{25}$ These estimated average cluster sizes for the different solvents, and their deuterated equivalents are summarised in Table 1 . The expansion conditions that were used to produce these clusters can be found in the ESI. $\dagger$

The $\mathrm{O}$ 1s Auger spectra of the different clusters and liquids, as well as their fully deuterated equivalents are presented in Fig. 2. The spectra feature a main peak at approximately $503 \mathrm{eV}$ and a pronounced shoulder at higher kinetic energy. On the lower kinetic energy side of the main peak a continuum of transitions that involve the inner-valence states can be found.

The differences between the Auger spectra of water, methanol and dimethyl ether have been discussed in detail for molecules in the gas- and amorphous solid phases by Rye et al., ${ }^{32}$ while the $\mathrm{O}$ 1s Auger of liquid water ${ }^{7}$ and methanol ${ }^{21}$ have also been discussed in previous publications, both in terms of their general Auger spectra and their respective proton dynamics. Generally, the spectral shape of the $\mathrm{O}$ 1s Auger spectra of gaseous water, methanol, and dimethyl ether have been found to be remarkably similar, such that their respective oxygen sites have been referred to as water-type structures. ${ }^{32}$ The $\mathrm{O} 1 \mathrm{~s}$ Auger spectra of the cluster phase can be seen in Fig. 2 and they very closely resemble the shape of their corresponding bulk liquid, ${ }^{7,21}$ with the main difference between the two spectra being the relative contribution of the high kinetic energy shoulder.

The Auger spectrum of these oxygen sites of water, methanol and dimethyl ether is characterised by a dominant feature at approximately $500 \mathrm{eV}$, followed by a progression of smaller peaks at gradually lower kinetic energy. There are, however, subtle differences in the spectra due to the presence of the $\mathrm{C}-\mathrm{O}$ bond in both methanol and dimethyl ether. Most importantly, in both methanol and dimethyl ether an additional peak is observed on the high kinetic energy side of the main peak. This means that in methanol and dimethyl ether the shoulder on the high kinetic energy side of the main peak is not exclusively due to non-local decay channels, as is the case in water, but rather contains a contribution from both local and non-local decay. This makes the determination of a relative propensity for local and non-local decay more complicated in methanol and dimethyl ether.

When comparing the different system sizes of water and methanol, namely small clusters, large clusters, and bulk liquid (see Fig. 3) it becomes apparent that the relative propensity for non-local decay, which contributes to the shoulder at $\sim 510 \mathrm{eV}$ kinetic energy, can be described as bulk-like for all studied systems. Within the accuracy of our data, the main deviation

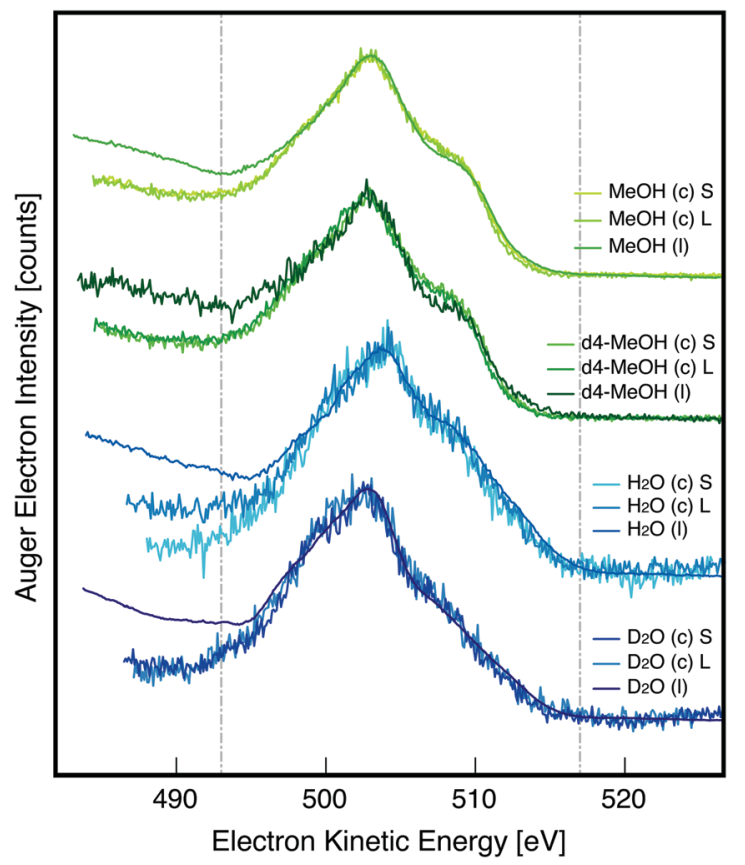

Fig. 3 Auger $\mathrm{O}$ 1s spectra of water $\left(\mathrm{H}_{2} \mathrm{O}\right)$, methanol $(\mathrm{MeOH})$ and their deuterated equivalents. The corresponding traces of small and large clusters as well as the bulk liquid are overlayed. The labels (c) and (l) are used to indicate cluster and liquid data respectively. The small and large cluster conditions are indicated by $\mathrm{S}$ and $\mathrm{L}$. The relative contribution of the local ( $503 \mathrm{eV}$ ) and the non-local decay (just below $510 \mathrm{eV}$ ) appear unaffected by the system size in all four compounds.

between the liquid phase and the cluster measurements is the increased inelastic scattering contribution to the continuum of transitions at lower kinetic energies $(<500 \mathrm{eV})$. This shows that in the systems measured here the relative propensity for the core-ionised excited state to decay via a non-local channel is not affected by the size and geometry. It is worth noting, that the small clusters studied here still contain a considerable number of monomer units on average ( $\sim 100$ molecules), at smaller sizes we would expect the non-local decay channel to decrease relative to the local channel, especially at sizes where no true bulk can be formed ( $<25$ molecules). ${ }^{1}$

Since the efficiency of non-local decay channels is determined by the distance to the nearest neighbour (the ICD rate is proportional to ${\frac{1}{r^{-6}}}^{33}$ ) and the number density of nearest neighbours, the relative contribution of this channel can vary significantly between e.g. bulk and surface regions. The main difference between molecules residing on the surface and in the bulk is that surface molecules have fewer nearest neighbours, but the distance to those neighbours can also vary compared with the bulk (as discussed earlier). The fact that we do not observe a change in the relative contribution of non-local channels when comparing clusters and the liquid is therefore taken as an indication that, within the probing depth of our experiment, the surface of the clusters and the liquid is largely similar in intermolecular distance. The strain that the smaller radius of curvature introduces to the cluster geometry might be 
compensated for by an increased internal pressure, which leads to bulk-like O-O distances. Note that a recent discussion of the inner valence ICD efficiency in water clusters ${ }^{34}$ does not apply here, as for core ionised systems the ICD channel is always open.

Similarly, the broadening we observe in the cluster phase Auger spectrum resembles the one observed in the respective liquid very closely rather than the sharp lineshape of the gas phase, ${ }^{32}$ as can be seen in Fig. 3. Such broadening effects of the cluster Auger spectrum of water have been discussed in detail by Öhrwall et al. ${ }^{15}$ Briefly, the broadening effect is related to the effect of structural heterogeneity on the polarisation screening of the charges created both in the intermediate $\mathrm{O} 1 \mathrm{~s}^{-1}$ state and the doubly valence ionised $\left(\mathrm{v}^{-2}\right)$ final states. Stronger intermolecular interactions give rise to greater inhomogeneous broadening because even small changes in structure lead to relatively large changes in intermolecular interaction energies. In addition, hydrogen bonds are more directional than other intermolecular interactions, so systems with more hydrogen bonds will be more sensitive to small structural changes. Conversely, weak intermolecular interactions mean that intermolecular interaction energies are relatively insensitive to structural changes, leading to reduced inhomogeneous broadening.

We now turn to the changes in the spectral shape of the Auger spectrum introduced by the substitution of deuterium for hydrogen. The effect of isotopic substitution can be seen most clearly in the case of liquid water, but the general trend is present in both the liquid and cluster system of water and methanol but not in the case of dimethyl ether. The deuterated systems show significantly less signal intensity in the spectral regions attributed to non-local decay, such as the shoulder at high kinetic energy. This loss of relative signal intensity is accompanied by an increase in signal contribution of the local decay region at the main peak, and on its low kinetic energy flank. This redistribution of signal intensity is attributed to proton dynamics in the core ionised intermediate state, as outlined earlier.

To separate the effects of proton dynamics from other changes in the spectrum, difference traces are formed by subtracting the spectrum of the deuterated species from its light equivalent. These difference traces are shown in Fig. 4(a). A positive area of the difference trace corresponds to a larger relative signal intensity in the light system, while a negative one corresponds to a higher signal intensity in the heavy system in that region. Interestingly, the difference traces of water and methanol have different shapes in the region above approximately $503 \mathrm{eV}$, with methanol showing a double peak structure while the difference trace of water only shows a single broad feature. The double peak structure of methanol can be attributed to the fact that the local Auger spectrum shows an additional feature on the high kinetic energy side of the main peak, whereas in water the main peak is the highest kinetic energy feature in the spectrum. ${ }^{32}$ The proton transfer then leads to a redistribution of the signal intensity in both of those peaks in methanol, which we observe as a double feature. It can already be seen in the difference traces that the size of the cluster modulates the amount of signal redistribution observed in the Auger region. In order to compare the isotope
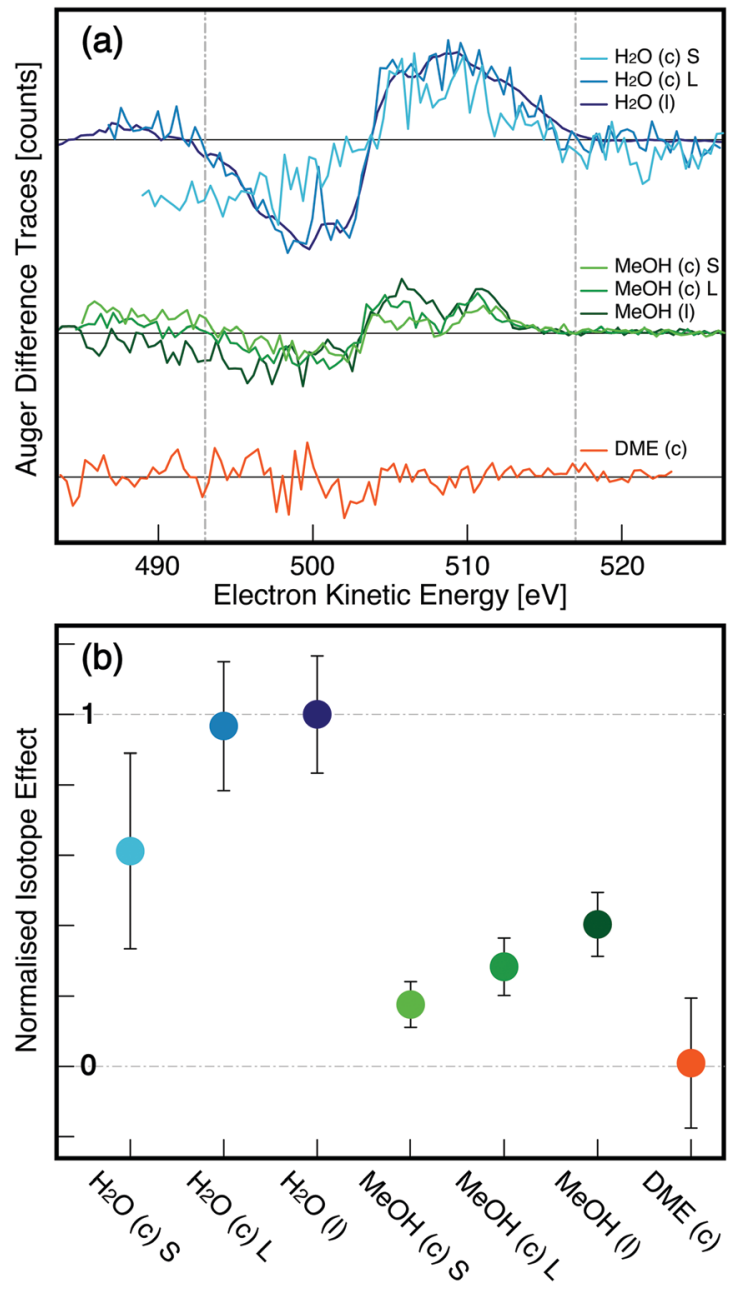

Fig. 4 (a) Normalised difference traces illustrating the redistribution of signal intensity due to isotopic substitution. (b) Total areas of the difference traces, normalised to liquid water. The dashed grey lines in panel (a) indicate the area used to normalise the spectra relative to one another. Cluster spectra are indicated by the label (c), and liquid spectra by (I). The relative cluster size is noted as $\mathrm{S}$ for small and $\mathrm{L}$ for large clusters.

effect in the different systems more clearly, we determined the integrated area under the difference traces and normalised them to liquid water, as shown in Fig. 4(b). These values express the isotope effect of each system as a fraction of the effect observed in liquid water. As outlined earlier, we interpret the relative propensity for proton dynamics, as observed by the isotope effect, as a measure of hydrogen bonding in the system.

Previous reports have shown that the bulk liquid of water and methanol both display a pronounced isotope effect, ${ }^{7,21}$ however the propensity of these effects has, to the best of our knowledge, not been compared directly. By comparing the normalised areas of the liquid-only Auger spectra of water and methanol, we find the isotope effect of liquid methanol to be reduced by a factor of $0.40(9)$ relative to liquid water. This indicates that the bulk intermolecular network in liquid methanol is approximately half as strong as the hydrogen bonding network found in liquid water. This reduction can be primarily attributed to the loss of $\mathrm{OH}$ groups, i.e. hydrogen bond donor sites, and thus a 
reduction in the possible number of hydrogen bonds formed per oxygen, since water can both accept and donate two hydrogen bonds per molecule, whereas methanol can only donate and accept one hydrogen bond per molecule. Any reduction beyond a factor of 0.5 is attributed to added steric strain imposed on the hydrogen bond network by the presence of the methyl groups.

As previously mentioned, all water and methanol systems presented here show an isotope effect in the O 1s Auger spectra. Such an isotope effect is absent in dimethyl ether, which is explained readily by the fact that there are no hydrogen bond donor sites located at the oxygen atom; the methyl groups effectively block the oxygen site in terms of hydrogen bond donation. However, it is reassuring that in a system without hydrogen bonds at the oxygen site, no isotope effect is observed in our measurements, indicating that artefacts are not introduced into the measurements or data handling procedure and to confirm the assumption that the absence of hydrogen bonding on the oxygen site leads to identical $\mathrm{O}$ 1s Auger spectra of the two isotopomers.

Returning to the case of water and methanol, the magnitude of the isotope effect observed in the cluster phase is found to decrease with decreasing system size, which we relate to a weakening of the overall hydrogen bond network as the system size is decreased. For water, the isotope effect of the large clusters is found to be $0.97(18)$ of the bulk value, while for the small clusters it is reduced to $0.61(28)$ of the bulk value. For methanol, the bulk value is $0.40(9)$ relative to liquid water, while for the large methanol clusters the effect was $0.28(8)$ and in small clusters it is further reduced to a fraction of $0.18(7)$ relative to bulk water. These relative propensities of proton dynamics can be seen in Fig. 4(b).

Overall, the isotope effect in water clusters is larger than in methanol clusters, following the effect observed in the bulk liquid; however, it is notable that, within the error bars, the isotope effect observed in small water clusters is stronger than, or at least comparable with, the effect found in liquid methanol. This indicates that the propensity for hydrogen bonding related proton dissociation is still higher in small water clusters than in bulk methanol.

Comparing the isotope effect for the large water clusters with the corresponding bulk liquid value, we find the observed effect in the clusters has converged to that of the bulk. For methanol, however, the isotope effect for the large clusters $(0.28(8))$ is still lower than the one observed in the bulk (0.40(9)). This could be due to the water hydrogen bond network converging to bulk values more quickly with size, or because the methanol clusters, despite showing a similar binding energy shift to water, are actually smaller than the water clusters produced in our experiment. When comparing the cluster and liquid measurements it is important to consider, that the Auger electrons still carry relatively low kinetic energy, $\sim 500 \mathrm{eV}$, the mean free path of the electrons in the liquid is $\sim 1-2 \mathrm{~nm} .^{7}$ This means that the Auger spectra contain contributions not only from the bulk but also a significant contribution from the surface layer and their relative contribution to the spectrum does not reflect the true surface-to-volume ratio.
For both water and methanol the isotope effect observed for small clusters is significantly smaller than that of the large clusters. To explain the difference in average strength of the hydrogen bond network for the two average cluster sizes, we first have to consider that the surface-to-volume ratio is higher in small clusters than in large clusters. Water molecules in the cluster surface layer form fewer hydrogen bonds on average than molecules in the bulk, therefore the propensity for proton dynamics is decreased at the surface. We estimate the surfaceto-volume ratio to be about 0.52 in our small water clusters and 0.37 in the large water clusters, assuming the clusters are structurally similar to low-density amorphous ice ${ }^{35}$ and spherical in shape.

The reduction in the isotope effect for smaller clusters is probably partly due to this effect; using the estimated size values given above, the surface-to-volume ratio of large clusters is 0.7 times that of the smaller clusters.

Due to the pronounced noise in the small water cluster measurement $( \pm 0.28)$, it is not possible from our work to determine to what degree the difference in the isotope effect between the small and large clusters is explained by the change in surface-to-volume ratio. Any difference exceeding the relative change in surface-tovolume ratio could be explained by reference to the intermolecular structure, and how the network is strained by confinement effects in the clusters.

The surface curvature will most likely also affect the intermolecular geometries at the water and methanol surfaces, but in a different manner. In the case of water, the surface molecules can no longer maintain a tetrahedral bonding environment, which causes a significant reduction in the number of hydrogen bonds each surface molecule can engage in, compared with the approximately four hydrogen bonds per molecule formed in the bulk. This leads to the formation of so-called dangling $\mathrm{OH}$ groups, ${ }^{3,36-38}$ which are unable to form hydrogen bonds and point towards the vacuum. Unsaturated water molecules are observed on all surfaces, but they are especially important to the overall properties of the cluster phase, due to their high surface-to-volume ratio. Any added deformation of the surface due to a reduction in the radius of curvature will affect the remaining few hydrogen bonds between the surface molecules, leading to a pronounced weakening of these interactions, a sketch of the flat and curved surface of water and methanol is shown in Fig. 5. The constituent molecules of the cluster would therefore attempt to counteract any stretching of intermolecular O-O distances, leading to a buildup of internal pressure within the cluster, which increases with decreasing cluster size and radius of curvature. ${ }^{39}$ Internal pressure deforms the hydrogen bond network in a way analogous to applying external pressure on the system, which has been shown to weaken hydrogen bonding. ${ }^{40}$ In the case of methanol, molecules at the interface arrange themselves with the methyl group pointing towards vacuum, ${ }^{5,6}$ which allows them to maintain a number of hydrogen bonds similar to that found in the bulk, as indicated in Fig. 5. If the curvature increases the spacing between those groups, the reduction of intermolecular interaction is relatively small, since the hydrophobic groups interact only weakly with one another. 
(a)

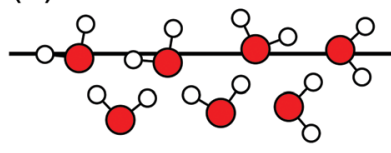

(c)

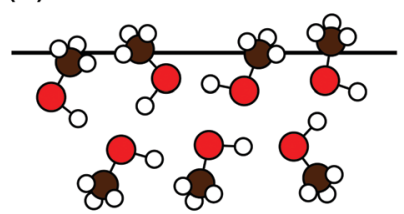

(b)

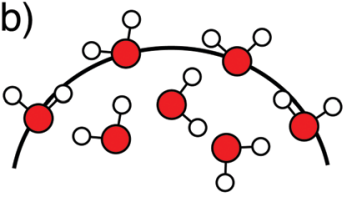

(d)

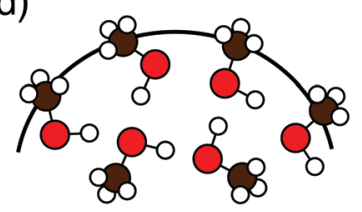

Fig. 5 Schematic comparing the flat and curved surface of water ((a) and (b)) and methanol ((c) and (d)) respectively. In the case of methanol the hydrophobic exclusion of the methyl group at the surface probably leads to decreased straining of the individual hydrogen bonds compared with water due to the curved surface geometry.

Another important aspect is that methanol only needs to form two hydrogen bonds per molecule to achieve a bulk-like hydrogen bond environment, as opposed to the four hydrogen bonds necessary for water, which is likely easier to achieve in both the bulk surface layer and in the cluster phase. The influence of the surface-to-volume ratio is therefore expected to be less pronounced than in water. Straining of the intermolecular structure due to confinement will, on the other hand, still exert a strong influence on the structure and in turn the hydrogen bonding, and since the hydrogen bond network counteracting deformation is weaker in methanol than in water (see bulk liquid values) this strain most likely deforms the network more strongly. This might in turn be related to the observation that the convergence to bulk behaviour occurs at larger cluster sizes for methanol than for water.

Irregular, i.e. non-spherical, shapes have been reported for water clusters in supersonic expansions. ${ }^{41}$ Such clusters usually form by the aggregation of smaller clusters in the expansion and will therefore show a larger surface-to-volume ratio, corresponding to those of the smaller clusters from which they derive. This leads to large clusters with a higher relative surface area, which would weaken the hydrogen-bond network further compared with their spherical siblings. Such shape effects would lead to a convergence to bulk at larger clusters, as the local molecular structure and surface-to-volume ratio may still resemble that of a smaller cluster.

\section{Conclusions}

We investigated the $\mathrm{O}$ 1s Auger spectra of molecular clusters consisting of water, methanol and dimethyl ether. Observing the size-dependence of the PTM-CS signal intensity - a spectral feature scaling with the hydrogen bond strength - we found the PTM-CS feature is present in all studied systems except dimethyl ether, which cannot donate a hydrogen bond at its oxygen site. The propensity for PTM-CS to occur is reduced in smaller clusters compared with the bulk for both water

and methanol, while the larger cluster case converges to bulk level in water but remains at intermediate values for methanol, which are not converged to the bulk. Assuming the average sizes of the clusters produced in our experiments are comparable for water and methanol, this indicates the hydrogen bond network converges to bulk more quickly in water than in methanol, and is more robust to structural strain, in terms of being able to form a strong hydrogen bond network even when the pronounced confinement effects of a small cluster geometry restrict the formation of a bulk-like network.

\section{Conflicts of interest}

There are no conflicts to declare.

\section{Acknowledgements}

The authors thank the Synchrotron SOLEIL (project number 20160555 and 20170443) and the Helmholtz-Zentrum Berlin (HZB) for the allocation of synchrotron radiation beamtime, and the local staff at both facilities for their support during the beamtime. In particular we acknowledge Aleksandar Milosavljevic, John Bozek and Emmanuel Robert for their help in the experimental execution of this work at the Pléiades Beamline, SOLEIL. Funding from the Swedish Research Council (VR) for the project VR 2018-00740 is acknowledged. CR and UH acknowledge funding by the Deutsche Forschungsgemeinschaft via Forschergruppe FOR1789. MP and MH acknowledge the support of the Academy of Finland. CC acknowledges program-oriented funds of the Helmholtz Association through the Center for FreeElectron Laser Science at Deutsches Elektronen-Synchrotron (DESY). CMS acknowledges funding from the Swedish Foundation for International Cooperation in Research and Higher Education (STINT).

\section{References}

1 U. Buck, C. C. Pradzynski, T. Zeuch, J. M. Dieterich and B. Hartke, Phys. Chem. Chem. Phys., 2014, 16, 6859.

2 K. R. Wilson, R. D. Schaller, D. T. Co, R. J. Saykally, B. S. Rude, T. Catalano and J. D. Bozek, J. Chem. Phys., 2002, 117, 7738-7744.

3 Q. Du, R. Superfine, E. Freysz and Y. R. Shen, Phys. Rev. Lett., 1993, 70, 2313-2316.

4 R. M. Townsend and S. A. Rice, J. Chem. Phys., 1991, 94, 2207-2218.

5 K. Wolfrum, H. Graener and A. Laubereau, Chem. Phys. Lett., 1993, 213, 41-46.

6 C. Stanners, Q. Du, R. Chin, P. Cremer, G. Somorjai and Y.-R. Shen, Chem. Phys. Lett., 1995, 232, 407-413.

7 S. Thürmer, M. Ončák, N. Ottosson, R. Seidel, U. Hergenhahn, S. E. Bradforth, P. Slavíček and B. Winter, Nature, Chemistry, 2013, 5, 590-596.

8 P. Slavíček, N. V. Kryzhevoi, E. F. Aziz and B. Winter, J. Phys. Chem. Lett., 2016, 7, 234-243. 
9 I. Unger, S. Thürmer, D. Hollas, E. F. Aziz, B. Winter and P. Slavíček, J. Phys. Chem. C, 2014, 118, 29142-29150.

10 I. Unger, D. Hollas, R. Seidel, S. Thürmer, E. F. Aziz, P. Slavíček and B. Winter, J. Phys. Chem. B, 2015, 119, 10750-10759.

11 M. O. Krause, J. Phys. Chem. Ref. Data, 1979, 8, 307-327.

12 C. Nicolas and C. Miron, J. Electron Spectrosc. Relat. Phenom., 2012, 185, 267-272.

13 P. Morin and C. Miron, J. Electron Spectrosc. Relat. Phenom., 2012, 185, 259-266.

14 L. S. Cederbaum, J. Zobeley and F. Tarantelli, Phys. Rev. Lett., 1997, 79, 4778-4781.

15 G. Öhrwall, R. F. Fink, M. Tchaplyguine, L. Ojamäe, M. Lundwall, R. R. T. Marinho, A. Naves de Brito, S. L. Sorensen, M. Gisselbrecht, R. Feifel, T. Rander, A. Lindblad, J. Schulz, L. J. Sæthre, N. Mårtensson, S. Svensson and O. Björneholm, J. Chem. Phys., 2005, 123, 054310.

16 T. Jahnke, H. Sann, T. Havermeier, K. Kreidi, C. Stuck, M. Meckel, M. Schöffler, N. Neumann, R. Wallauer, S. Voss, A. Czasch, O. Jagutzki, A. Malakzadeh, F. Afaneh, T. Weber, H. Schmidt-Böcking and R. Dörner, Nat. Phys., 2010, 6, 139-142.

17 M. Mucke, M. Braune, S. Barth, M. Förstel, T. Lischke, V. Ulrich, T. Arion, U. Becker, A. Bradshaw and U. Hergenhahn, Nat. Phys., 2010, 6, 143-146.

18 J. Zobeley, R. Santra and L. S. Cederbaum, J. Chem. Phys., 2001, 115, 5076-5088.

19 I. B. Müller and L. S. Cederbaum, J. Chem. Phys., 2005, 122, 094305.

20 R. Sankari, M. Ehara, H. Nakatsuji, Y. Senba, K. Hosokawa, H. Yoshida, A. De Fanis, Y. Tamenori, S. Aksela and K. Ueda, Chem. Phys. Lett., 2003, 380, 647-653.

21 C.-M. Saak, I. Unger, B. Brena, C. Caleman and O. Björneholm, Phys. Chem. Chem. Phys., 2019, 21, 15478-15486.

22 Y. I. Neela, A. S. Mahadevi and G. N. Sastry, J. Phys. Chem. B, 2010, 114, 17162-17171.

23 A. Lindblad, J. Söderström, C. Nicolas, E. Robert and C. Miron, Rev. Sci. Instrum., 2013, 84, 113105.
24 S. Barth, M. Ončák, V. Ulrich, M. Mucke, T. Lischke, P. Slavíček and U. Hergenhahn, J. Phys. Chem. A, 2009, 113, 13519-13527.

25 B. L. Yoder, A. H. C. West, B. Schläppi, E. Chasovskikh and R. Signorell, J. Chem. Phys., 2013, 138, 044202.

26 O. F. Hagena, J. Chem. Phys., 1972, 56, 1793.

27 O. F. Hagena, Surf. Sci., 1981, 106, 101-116.

28 C. Bobbert, S. Schütte, C. Steinbach and U. Buck, Eur. Phys. J. D, 2002, 19, 183-192.

29 R. Seidel, M. N. Pohl, H. Ali, B. Winter and E. F. Aziz, Rev. Sci. Instrum., 2017, 88, 073107.

30 T. Kachel, Journal of Large-Scale Research Facilities, 2016, 2, A72.

31 T. E. Gartmann, S. Hartweg, L. Ban, E. Chasovskikh, B. L. Yoder and R. Signorell, Phys. Chem. Chem. Phys., 2018, 20, 16364-16371.

32 R. R. Rye, T. E. Madey, J. E. Houston and P. H. Holloway, J. Chem. Phys., 1978, 69, 1504-1512.

33 R. Santra and L. S. Cederbaum, Phys. Rep., 2002, 368, 1-117. 34 C. Richter, D. Hollas, C.-M. Saak, M. Förstel, T. Miteva, M. Mucke, O. Björneholm, N. Sisourat, P. Slavíček and U. Hergenhahn, Nat. Commun., 2018, 9, 4988.

35 R. Martoňák, D. Donadio and M. Parrinello, J. Chem. Phys., 2005, 122, 134501.

36 Q. Du, E. Freysz and Y. R. Shen, Science, 1994, 264, 826-828. 37 I. V. Stiopkin, C. Weeraman, P. A. Pieniazek, F. Y. Shalhout, J. L. Skinner and A. V. Benderskii, Nature, 2011, 474, 192-195.

38 I.-F. W. Kuo, Science, 2004, 303, 658-660.

39 B. C. Garrett, S. M. Kathmann and G. K. Schenter, in Thermochemistry and Kinetics of Evaporation and Condensation for Small Water Clusters, ed. V. Buch and J. P. Devlin, Springer Berlin Heidelberg, Berlin, Heidelberg, 2003, pp. 25-51.

40 A. K. Soper and M. A. Ricci, Phys. Rev. Lett., 2000, 84, 2881-2884.

41 J. Lengyel, A. Pysanenko, V. Poterya, P. Slavíček, M. Fárník, J. Kočišek and J. Fedor, Phys. Rev. Lett., 2014, 112, 113401. 